SOGANG-HEP 302/03

\title{
Complete BFT Embedding of Massive Theory with One- and Two-form Gauge Fields
}

\author{
Seung-Kook Kim ${ }^{1}$, Yong-Wan Kim ${ }^{2}$ and Young-Jai Park ${ }^{3}$ \\ ${ }^{1}$ Department of Physics, Seonam University, \\ Namwon, Chonbuk 590-170, Korea \\ ${ }^{2}$ Department of Physics and Institute for Science and Technology, \\ Sejong University, Seoul 143-747, Korea \\ ${ }^{3}$ Department of Physics and Basic Science Research Institute, \\ Sogang University, C.P.O. Box 1142, Seoul 100-611, Korea
}

(December 24, 2018)

\begin{abstract}
We study the constraint structure of the topologically massive theory with one- and two-form fields in the framework of BatalinFradkin-Tyutin embedding procedure. Through this analysis we obtain a new type of Wess-Jumino action with novel symmetry, which is originated from the topological coupling term, as well as the Stückelberg action related to the explicit gauge breaking mass terms from the original theory.
\end{abstract}

PACS: 11.10.Ef, 11.30.Ly, 11.15.-q

Keywords: Topological mass generation; Hamiltonian and Lagrangian embedding; Antisymmetric tensor gauge fields

\footnotetext{
${ }^{1}$ Electronic address: skandjh@empal.com

${ }^{2}$ Electronic address: ywkim65@netian.com

${ }^{3}$ Electronic address: yjpark@ccs.sogang.ac.kr
} 


\section{Introduction}

Dirac scheme in the Hamiltonian formalism [1] has been widely used to quantize second-class constraint system in which the Poisson brackets of constraints do not vanish on constraint surface. For second-class constraint system, however, there are under unfavorable circumstances in finding canonically conjugate pairs since the resulting Dirac brackets may be in general field-dependent and/or nonlocal, and have a serious ordering problem between field operators. On the other hand, the quantization of first-class constraint system $[2,3]$ has been well appreciated in a gauge invariant manner preserving Becci-Rouet-Stora-Tyutin symmetry [4]. Therefore, if secondclass constraint system can be converted into first-class one using auxiliary degrees of freedom to extend a phase space, we do not actually need to define Dirac brackets and then the remaining quantization program follows the method of Ref. [2, 3, 4]. This procedure has been established by Batalin, Fradkin, and Tyutin (BFT) $[5,6]$ and extensively studied in the canonical formalism for various models including massive gauge fields [7], spontaneously broken gauge theories [8], $S U(2) / S U(3)$ Skyrmion models [9], non-linear sigma models [10], noncommutative systems [11], and many others [12]. The BFT embedding formalism has been also widely applied to obtain the WessZumino (WZ) actions [13] showing that original theory can be regarded as a gauge-fixed version of extended gauge system, while verifying dual equivalent descriptions [14] in particular gauges from the phase space partition function corresponding to the BFT embedded involutive Hamiltonian.

On the other hand, antisymmetric tensor fields appearing first as a mediator of the interaction [15] have been much interested in as an alternative of the Higgs mechanism without residual Higgs scalar $[16,17]$. With the topologically interacting terms of the form $B \wedge F$, this mechanism is considered generic in string phenomenology [18]. Moreover, various dual descriptions between different models have been widely studied where antisymmetric tensor fields play an important role in realization of dualities [19, 20]. In particular, several years ago Banerjee and Banerjee had studied a master Lagrangian [21] which is a first order massive spin-one theory involving antisymmetric tensor fields in order to show the dual equivalence of the Proca model and the massive Kalb-Ramond model within a path integral framework. Recently, Harikumar and Sivakumar [22] have elaborated on the Lagrangian through the Hamiltonian and Lagrangian embedding technique, and have 
shown that the embedded theory with appropriate gauge fixing is equivalent to the $B \wedge F$ theory on the level of Hamiltonian. The Lagrangian regarded as a topologically massive theory has interesting constraint structure related to the topological coupling term as well as the gauge symmetry breaking mass term. As results, the second-class constraints appears from two different origins: One comes from the explicit gauge symmetry breaking mass term, and the other from the topological coupling term. Since this theory have been studied by a reduction of the set of constraints through FaddeevJackiw method [23], there still remains to be clarified the role of auxiliary fields, which are originated from the topological interaction term.

In the present paper, we shall fully apply the BFT method to the topologically massive theory with one- and two-form gauge fields. As results, we will explicitly show that auxiliary fields in part for the BFT embedding are nothing but the well-known Stückelberg fields on one hand, and find a new type of WZ action [26] composed of the remaining auxiliary fields, which are originated from symplectic structure of the model on the other hand. We also clarify the underlying constraint structure concerning irreducible and reducible constraints.

In section 2, we newly construct the constraint structure of this model, which is much simpler than that of the previous work [22] due to the absence of derivatives in their Poisson brackets. In section 3, we carry out the complete BFT embedding of the theory which has local gauge symmetry supplemented with other: Local gauge symmetry is recovered from explicitly gauge symmetry breaking mass terms and the other comes from underlying symplectic structure of topological term. In section 4 , by identifying new auxiliary degrees of freedom with Stückelberg vector fields and new type of WZ fields, we obtain simultaneously the Stückelberg Lagrangian related to the explicit gauge breaking mass terms and a new type of WZ action with novel symmetry, which is originated from the symplectic structure of the theory. In section 5, we revisit the original theory making use of gauging technique to show the equivalence of the gauged Lagrangian and the Stückelberg Lagrangian on the constraint surface. Conclusion is devoted in section 6 . 


\section{Constraint Structure of Topologically Mas- sive Theory}

In this section, we consider topologically massive theory with one- and twoform fields, described by the following first order Lagrangian [21, 22]:

$$
\mathcal{L}=-\frac{1}{4} B_{\mu \nu} B^{\mu \nu}+\frac{1}{2} A_{\mu} A^{\mu}+\frac{1}{2 m} \epsilon_{\mu \nu \rho \sigma} B^{\mu \nu} \partial^{\rho} A^{\sigma} .
$$

From the symmetrized form of the Lagrangian of

$$
\mathcal{L}=-\frac{1}{4} B_{\mu \nu} B^{\mu \nu}+\frac{1}{2} A_{\mu} A^{\mu}+\frac{1}{4 m} \epsilon_{\mu \nu \rho \sigma} B^{\mu \nu} \partial^{\rho} A^{\sigma}-\frac{1}{4 m} \epsilon_{\mu \nu \rho \sigma} \partial^{\mu} B^{\nu \rho} A^{\sigma}
$$

we read the canonical momenta as

$$
\begin{aligned}
& \pi_{0}=0, \quad \pi_{i}=\frac{1}{4 m} \epsilon_{i j k} B^{j k}, \\
& \pi_{0 i}=0, \quad \pi_{i j}=-\frac{1}{2 m} \epsilon_{i j k} A^{k},
\end{aligned}
$$

where we denote $\epsilon_{0 i j k}=\epsilon_{i j k}$ and $\epsilon^{123}=+1$. Then, the primary Hamiltonian yields

$$
\mathcal{H}_{p}=\mathcal{H}_{c}+\lambda^{0} \pi_{0}+\lambda^{i} \Omega_{i}+\Sigma^{0 i} \pi_{0 i}+\Sigma^{i j} \Omega_{i j}
$$

with the Lagrange multipliers $\lambda^{0}, \lambda^{i}, \Sigma^{0 i}$, and $\Sigma^{i j}$, where the canonical Hamiltonian is given by

$$
\begin{aligned}
\mathcal{H}_{c} & =\frac{1}{4} B_{i j} B^{i j}-\frac{1}{2} A_{i} A^{i}+\frac{1}{2} B_{0 i} B^{0 i}-\frac{1}{2} A_{0} A^{0} \\
& -\frac{1}{m} \epsilon^{i j k} B_{0 i} \partial_{j} A_{k}-\frac{1}{2 m} A^{0} \epsilon^{i j k} \partial_{i} B_{j k},
\end{aligned}
$$

and the primary constraints are defined as

$$
\begin{aligned}
& \pi_{0} \approx 0, \quad \Omega_{i} \equiv \pi_{i}-\frac{1}{4 m} \epsilon_{i j k} B^{j k} \approx 0 \\
& \pi_{0 i} \approx 0, \quad \Omega_{i j} \equiv \pi_{i j}+\frac{1}{2 m} \epsilon_{i j k} A^{k} \approx 0 .
\end{aligned}
$$

From the time stability conditions of the constraints $\pi_{0}$ and $\pi_{0 i}$, we have obtained two additional secondary constraints as

$$
\begin{aligned}
\Lambda & \equiv \dot{\pi}_{0}=\left\{\pi_{0}, \mathcal{H}_{p}\right\}=A_{0}+\frac{1}{2 m} \epsilon^{i j k} \partial_{i} B_{j k} \approx 0 \\
\Lambda_{i} & \equiv \dot{\pi}_{0 i}=\left\{\pi_{0 i}, \mathcal{H}_{p}\right\}=-B_{0 i}+\frac{1}{m} \epsilon_{i j k} \partial^{j} A^{k} \approx 0,
\end{aligned}
$$


and the constraints $\Omega_{i}, \Omega_{i j}$ fix the Lagrange multipliers $\Sigma^{i j}, \lambda^{i}$, respectively. The other Lagrange multipliers $\lambda^{0}, \Sigma^{0 i}$ are also determined by requiring consistency of the secondary constraints $\Lambda, \Lambda_{i}$ with the equations of motion, and thus no further new constraints are generated. As a result, the Poisson brackets of all the constraints (2.6) and (2.7) are obtained as

$$
\begin{aligned}
\left\{\pi_{0}, \Lambda\right\} & =-\delta(x-y), \\
\left\{\pi_{0 i}, \Lambda_{j}\right\} & =\delta_{i j} \delta(x-y), \\
\left\{\Omega_{i}, \Omega_{j k}\right\} & =-\frac{1}{m} \epsilon_{i j k} \delta(x-y), \\
\left\{\Omega_{i}, \Lambda_{j}\right\} & =\frac{1}{m} \epsilon_{i j k} \partial_{x}^{k} \delta(x-y), \\
\left\{\Omega_{i j}, \Lambda\right\} & =\frac{1}{m} \epsilon_{i j k} \partial_{x}^{k} \delta(x-y)
\end{aligned}
$$

showing that the constraint structure of the topologically massive theory be fully second-class.

Now, instead of reducing the constraints $\Omega_{i}, \Omega_{i j}$ strongly by making use of Faddeev-Jackiw scheme [23] and considering only gauge degrees of freedom as done in the previous work [22], we will keep the full set of the constraints (2.6) and (2.7). In order for studying the whole constraints efficiently, we further need to modify the constraints (2.7) as

$$
\begin{aligned}
\Lambda^{\prime} & \equiv \partial^{i} \Omega_{i}+\Lambda \\
& =\partial^{i} \pi_{i}+A^{0}+\frac{1}{4 m} \epsilon^{i j k} \partial_{i} B_{j k} \approx 0, \\
\Lambda_{i}^{\prime} & \equiv \partial^{j} \Omega_{i j}-\Lambda_{i} \\
& =\partial^{j} \pi_{i j}+B_{0 i}-\frac{1}{2 m} \epsilon_{i j k} \partial^{j} A^{k} \approx 0,
\end{aligned}
$$

which are equivalent to the original ones, $\Lambda, \Lambda_{i}$ on the constraint surface. Then, the set of these new constraints makes the constraint algebra (2.8) much simpler and concise as

$$
\begin{aligned}
\left\{\pi_{0}, \Lambda^{\prime}\right\} & =-\delta(x-y) \\
\left\{\pi_{0 i}, \Lambda_{j}^{\prime}\right\} & =\delta_{i j} \delta(x-y) \\
\left\{\Omega_{i}, \Omega_{j k}\right\} & =-\frac{1}{m} \epsilon_{i j k} \delta(x-y),
\end{aligned}
$$


while the others identically vanish ${ }^{4}$.

As results, we have obtained the fully second-class constraints (2.6) and (2.9) for the first order Lagrangian of the topologically massive theory. Notice that this new algebra contains no derivatives unlikely in Eq. (2.8) as well as vanishing brackets between the constraints $\Omega_{i}, \Omega_{i j}$ and $\Lambda, \Lambda_{i}$. In fact, due to the absence of the derivatives in the new Poisson brackets, one can easily convert the second-class constraints (2.6) and (2.9) into corresponding first-class ones making use of the BFT embedding technique, which we will explicitly study in the next section. If we use the constraint algebra as it stands in Eq. (2.8) containing the derivatives, BFT embedded constraints and fields may have non-local expressions which would make the quantization intractable.

On the other hand, making use of the definition of the Dirac brackets as

$$
\begin{aligned}
\{A(x), B(y)\}_{D B} & =\{A(x), B(y)\}_{P B} \\
& -\int d w d z\left\{A(x), \phi_{\alpha}(w)\right\} C^{\alpha \beta}(w, z)\left\{\phi_{\beta}(z), B(y)\right\}
\end{aligned}
$$

where the matrix $C^{\alpha \beta}$ is an inverse of $\left\{\phi_{\alpha}(x), \phi_{\beta}(y)\right\}=C_{\alpha \beta}(x, y)$ along with the constraints denoted by $\phi_{\alpha}=\left(\pi_{0}, \pi_{0 i}, \Lambda, \Lambda_{i}, \Omega_{i}, \Omega_{i j}\right)$, we have obtained the following non-vanishing Dirac Brackets

$$
\begin{aligned}
\left\{A^{0}(x), A^{i}(y)\right\}_{D} & =\partial_{x}^{i} \delta(x-y), \\
\left\{A^{0}(x), \pi_{i j}(y)\right\}_{D} & =-\frac{1}{2 m} \epsilon_{i j k} \partial_{x}^{k} \delta(x-y), \\
\left\{A^{i}(x), \pi_{j}(y)\right\}_{D} & =\frac{1}{2} \delta_{j}^{i} \delta(x-y), \\
\left\{A^{i}(x), B^{j k}(y)\right\}_{D} & =-m \epsilon^{i j k} \delta(x-y), \\
\left\{\pi_{i}(x), B_{0 j}(y)\right\}_{D} & =\frac{1}{2 m} \epsilon_{i j k} \partial_{x}^{k} \delta(x-y), \\
\left\{\pi_{i}(x), \pi_{j k}(y)\right\}_{D} & =\frac{1}{4 m} \epsilon_{i j k} \delta(x-y), \\
\left\{B^{0 i}(x), B^{j k}(y)\right\}_{D} & =\left(\delta^{i j} \partial_{x}^{k}-\delta^{i k} \partial_{x}^{j}\right) \delta(x-y), \\
\left\{B^{i j}(x), \pi_{k l}(y)\right\}_{D} & =\frac{1}{2}\left(\delta_{k}^{i} \delta_{l}^{j}-\delta_{k}^{j} \delta_{l}^{i}\right) \delta(x-y)
\end{aligned}
$$

\footnotetext{
${ }^{4}$ From now on, we will omit the prime symbols on the constraints $\Lambda, \Lambda_{i}$
} 
in order to compare with the results obtained from the BFT embedding which automatically leads to the Dirac brackets at the level of Poisson brackets in the extended phase space.

It seems appropriate to comment on the constraints $\Omega_{i}$, and $\Omega_{i j}$. These constraints come from the topological term in the Lagrangian (2.1). In the scheme of Faddeev-Jackiw quantization [23] which deals with only the dynamical degrees of freedom, these could be eliminated from the start while modifying other brackets of the fields. Harikumar and Sivakumar [22] has worked in this direction. However, in the present paper, we will keep these constraints with the others as it stands and embed the whole constraints into much larger phase space than their phase space, which we mean complete BFT embedding. This will give a new type of WZ action with novel symmetry as well as the Stückelberg action with the usual gauge symmetry.

\section{Complete BFT Hamiltonian Embedding}

Since we know how to quantize first-class constraint system very well while second-class system may have serious ordering and/or non-local problems, it is preferred to deal with first-class constraint system. The BFT embedding prescription makes in a systematic way second-class constraint Hamiltonian system into corresponding first-class one. In order for that purpose, we introduce auxiliary fields having involutive relations in which not only modified new constraints in the enlarged space are strongly vanishing with each other but also they have vanishing Poisson brackets, not the Dirac brackets, with physical quantities such as Hamiltonian and fields themselves. Here, "physical' means gauge invariant since resulting modified new quantities would be first-class by construction. Practically, with the aid of auxiliary fields $\Phi^{\alpha}$, one for each constraint, satisfying with

$$
\left\{\Phi^{\alpha}(x), \Phi^{\beta}(y)\right\}=\omega^{\alpha \beta}(x, y),
$$

while vanishing with the original fields, we construct the involutive relations as

$$
\begin{aligned}
\left\{\tilde{\varphi}_{\alpha}(x), \tilde{\varphi}_{\beta}(y)\right\} & =0, \\
\left\{\tilde{\varphi}_{\alpha}(x), \tilde{\mathcal{F}}(y)\right\} & =0,
\end{aligned}
$$


where the new constraints $\tilde{\varphi}_{\alpha}$, and physical quantities $\tilde{\mathcal{F}}$ are given by $\tilde{\varphi}_{\alpha} \sim$ $\phi_{\alpha}+\sum_{n=1}\left(\Phi^{\alpha}\right)^{n}$, and $\tilde{\mathcal{F}} \sim \mathcal{F}+\sum_{n=1}\left(\Phi^{\alpha}\right)^{n}$, respectively, with appropriate coefficients in front of $\left(\Phi^{\alpha}\right)^{n}$. In case we set the auxiliary fields $\Phi^{\alpha}$ to be zero, those quantities are reduced to the original ones, i.e., $\left.\tilde{\varphi}_{\alpha}\right|_{\Phi^{\alpha}=0}=\phi_{\alpha}$ (or, $\left.\tilde{\mathcal{F}}\right|_{\Phi^{\alpha}=0}=\mathcal{F}$ ).

Now, let us explicitly solve the involutive relations, Eqs. (3.2) and (3.3), by making use of the following Ansatz of

$$
\varphi_{\alpha}^{(1)}(x)=\int d y X_{\alpha \beta}(x, y) \Phi^{\beta}(y)
$$

Inserting the Ansatz to the involutive relations between the constraints (3.2), we obtain in the zeroth order of the auxiliary fields $\Phi^{\alpha}$ as

$$
\begin{aligned}
0 & =\left\{\varphi_{\alpha}, \varphi_{\beta}\right\}_{\mathcal{O}}+\left\{\varphi_{\alpha}^{(1)}, \varphi_{\beta}^{(1)}\right\}_{\Phi} \\
& =C_{\alpha \beta}(x, y)+\int d w d z X_{\alpha \gamma}(x, w) \omega^{\gamma \delta}(w, z) X_{\delta \beta}(z, y)
\end{aligned}
$$

and in the first order of $\Phi^{\alpha}$ as

$$
\left\{\varphi_{\alpha}, \varphi_{\beta}^{(1)}\right\}_{\mathcal{O}}+\left\{\varphi_{\alpha}^{(1)}, \varphi_{\beta}\right\}_{\mathcal{O}}+\left\{\varphi_{\alpha}^{(1)}, \varphi_{\beta}^{(2)}\right\}_{\Phi}+\left\{\varphi_{\alpha}^{(2)}, \varphi_{\beta}^{(1)}\right\}_{\Phi}=0
$$

and so on. Here, the subscript $\mathcal{O}($ or, $\Phi)$ denotes the Poisson bracket with respect to the original variables $(q, p)$ (or, the auxiliary fields, $\Phi)$. Then, by knowing the first order correction $\varphi_{\alpha}^{(1)}$, we can obtain the next order $\varphi_{\alpha}^{(2)}$ from Eq. (3.6), and these procedure continue iteratively until we determine involutive new constraints completely.

Through the similar steps as above, we can also get the first order correction for physical quantity as

$$
\mathcal{F}^{(1)}(x)=-\int d y d z d w \Phi^{\alpha}(x) \omega_{\alpha \beta}(x, y) X^{\beta \gamma}(y, z)\left\{\varphi_{\gamma}(z), A(w)\right\}
$$

in the zeroth order of the auxiliary fields $\Phi^{\alpha}$. This correction has been obtained explicitly from the ansatz (3.4) and the involutive relation (3.3). Also, higher order corrections $\mathcal{F}^{(n)}(n \geq 2)$ can be obtained iteratively from Eq. (3.3) to find completely involutive quantities out along with the modified new constraints.

It is appropriate to comment on the choice of $\omega^{\alpha \beta}$ and $X_{\alpha \beta}$ which may be the functions of the original variables. Although there are no known criteria 
to best choice $\omega^{\alpha \beta}$ and $X_{\alpha \beta}$, but final forms of the modified constraints and the physical quantities depend on what the matrix elements of $\omega^{\alpha \beta}$ and $X_{\alpha \beta}$ are. Nevertheless, among different expressions of constraints and physical quantities obtained from choosing any set of $\omega^{\alpha \beta}$ and $X_{\alpha \beta}$, they are related to each other with canonical transformations.

Now return to our model. By following the above BFT prescription, we introduce auxiliary fields paired as $\left(\theta, \pi_{\theta}\right),\left(Q^{i}, P_{i}\right)$, and $\left(\Phi^{i}, \Phi^{j k}\right)$ :

$$
\Phi^{\alpha}=\left(\theta, Q^{i}, \pi_{\theta}, P^{i}, \Phi^{i}, \Phi^{i j}\right)
$$

which correspond to the constraints $\phi_{\alpha}=\left(\pi_{0}, \pi_{0 i}, \Lambda, \Lambda_{i}, \Omega_{i}, \Omega_{i j}\right)$, respectively. Without any loss of generality, let us for simplicity make a proper choice for the auxiliary fields canonically conjugated as follows

$$
\left\{\Phi^{\alpha}(x), \Phi^{\beta}(y)\right\}=\omega^{\alpha \beta}(x, y)=\left(\begin{array}{cccccc}
0 & 0 & 1 & 0 & 0 & 0 \\
0 & 0 & 0 & \delta^{i j} & 0 & 0 \\
-1 & 0 & 0 & 0 & 0 & 0 \\
0 & -\delta^{i j} & 0 & 0 & 0 & 0 \\
0 & 0 & 0 & 0 & 0 & \epsilon^{i j k} \\
0 & 0 & 0 & 0 & -\epsilon^{i j k} & 0
\end{array}\right) \delta(x-y)
$$

Here, note that the auxiliary fields $\Phi^{i}, \Phi^{i j}$ in the last two columns is explicitly given by

$$
\left\{\Phi^{i}(x), \Phi^{j k}(y)\right\}=\epsilon^{i j k} \delta(x-y),
$$

which choice makes it possible to embed the constraints originated from the topological term. With the above choice of $\omega^{\alpha \beta}$, we solve the Eq. (3.5) and find a simple solution as

$$
X_{\alpha \beta}(x, y)=\left(\begin{array}{cccccc}
1 & 0 & 0 & 0 & 0 & 0 \\
0 & \delta^{i j} & 0 & 0 & 0 & 0 \\
0 & 0 & 1 & 0 & 0 & 0 \\
0 & 0 & 0 & \delta^{i j} & 0 & 0 \\
0 & 0 & 0 & 0 & I_{3 \times 3} & 0 \\
0 & 0 & 0 & 0 & 0 & \frac{1}{m} I_{3 \times 3}
\end{array}\right) \delta(x-y)
$$

where $I_{3 \times 3}$ denotes a $3 \times 3$ identity matrix. 
As results, we have obtained the strongly involutive new constraints from Eq. (3.4) as

$$
\begin{aligned}
\tilde{\pi}_{0} & =\pi_{0}+\theta, \quad \widetilde{\pi}_{0 i}=\pi_{0 i}+Q_{i} \\
\widetilde{\Lambda} & =\Lambda+\pi_{\theta}, \quad \widetilde{\Lambda}_{i}=\Lambda_{i}+P_{i} \\
\widetilde{\Omega}_{i} & =\Omega_{i}+\Phi_{i}, \quad \widetilde{\Omega}_{i j}=\Omega_{i j}+\frac{1}{m} \Phi_{i j}
\end{aligned}
$$

with no higher order contributions of the auxiliary fields due to our proper choice of $\omega^{\alpha \beta}$ and $X_{\alpha \beta}$ while satisfying the involutive relation (3.2). Moreover, from the inverse matrix $\omega_{\alpha \beta}$ and $X^{\alpha \beta}$ and the solution of the physical fields (3.7), we have also gotten the strongly involutive physical fields [25] as follows

$$
\begin{aligned}
\widetilde{A}^{0} & =A^{0}+\pi_{\theta}, \\
\widetilde{A}^{i} & =A^{i}+\partial^{i} \theta-\frac{1}{2} \epsilon^{i j k} \Phi_{j k}, \\
\widetilde{\pi}_{0} & =\pi_{0}+\theta \\
\widetilde{\pi}_{i} & =\pi_{i}-\frac{1}{2 m} \epsilon_{i j k} \partial^{j} Q^{k}+\frac{1}{2} \Phi_{i}, \\
\widetilde{B}^{0 i} & =B^{0 i}+P^{i}, \\
\widetilde{B}^{i j} & =B^{i j}-\left(\partial^{i} Q^{j}-\partial^{j} Q^{i}\right)+m \epsilon^{i j k} \Phi_{k}, \\
\widetilde{\pi}_{0 i} & =\pi_{0 i}+Q_{i}, \\
\widetilde{\pi}_{i j} & =\pi_{i j}-\frac{1}{2 m} \epsilon_{i j k} \partial^{k} \theta+\frac{1}{2 m} \Phi_{i j}
\end{aligned}
$$

satisfying the involutive relation (3.3). Note that all the physical fields are terminated in the first order of the auxiliary fields of the BFT embedding sequence and there are no higher order contributions. One can easily checked that the Poisson brackets of Eq. (3.12) in the extended phase space give exactly the same Dirac brackets in the original phase space as given in Eq. (2.11) as they should be.

On the other hand, canonical Hamiltonian in the extended phase space can be obtained either by solving the strongly involutive relation (3.3) in replacement of $\tilde{\mathcal{F}}$ with a Hamiltonian function or by using the physical fields (3.12) in the canonical Hamiltonian (2.5) written by the tilde fields. Since these methods of obtaining canonical Hamiltonian are known to be equivalent to each other on the constraint surface, which are weakly equivalent, we will 
follow the latter approach. Then, the canonical Hamiltonian (2.5) is simply written in the extended phase space as follows

$$
\begin{aligned}
\widetilde{\mathcal{H}}_{c} & =\frac{1}{4}\left(B_{i j}-Q_{i j}\right)^{2}+\frac{1}{2} m \epsilon_{i j k}\left(B^{i j}-Q^{i j}\right) \Phi^{k}-\frac{1}{2} m^{2} \Phi_{i} \Phi^{i} \\
& -\frac{1}{2}\left(A_{i}+\partial_{i} \theta\right)^{2}+\frac{1}{2} \epsilon_{i j k}\left(A^{i}+\partial^{i} \theta\right) \Phi^{j k}+\frac{1}{4} \Phi_{i j} \Phi^{i j} \\
& +\frac{1}{2}\left(B_{0 i}+P_{i}\right)^{2}-\frac{1}{m} \epsilon_{i j k}\left(B^{0 i}+P^{i}\right) \partial^{j} A^{k}-\frac{1}{m}\left(B_{0 i}+P_{i}\right) \partial_{j} \Phi^{i j} \\
& -\frac{1}{2}\left(A_{0}+\pi_{\theta}\right)^{2}-\frac{1}{2 m}\left(A_{0}+\pi_{\theta}\right) \epsilon_{i j k} \partial^{i} B^{j k}+\left(A_{0}+\pi_{\theta}\right) \partial_{i} \Phi^{i}
\end{aligned}
$$

where $Q_{i j}$ denote $Q_{i j}=\partial_{i} Q_{j}-\partial_{j} Q_{i}$. By construction, this canonical Hamiltonian satisfies the strongly involutive relations with the modified constraints $\tilde{\varphi}_{\alpha}$, i.e., $\left\{\tilde{\varphi}_{\alpha}, \widetilde{\mathcal{H}}_{c}\right\}=0$. This ends the BFT embedding for the topologically massive theory with the one- and two-form fields.

\section{Corresponding Lagrangian with new type of WZ term}

In this section, we find the corresponding Lagrangian of the extended canonical Hamiltonian by making use of the phase space path integral. For this purpose we modify further the canonical Hamiltonian (3.13) to an equiv-

alent one on the constraint surface which generates the Gauss' constraints naturally. The equivalent Hamiltonian given by

$$
\widetilde{\mathcal{H}}_{c}^{\prime}=\widetilde{\mathcal{H}}_{c}+\pi_{\theta} \widetilde{\Lambda}+P_{i} \widetilde{\Lambda}_{i}
$$

yields the Gauss' constraints as

$$
\begin{aligned}
\frac{d}{d t} \widetilde{\pi}_{0} & =\left\{\widetilde{\pi}_{0}, \widetilde{\mathcal{H}}_{c}^{\prime}\right\}=\widetilde{\Lambda}, \\
\frac{d}{d t} \widetilde{\pi}_{0 i} & =\left\{\widetilde{\pi}_{0 i}, \widetilde{\mathcal{H}}_{c}^{\prime}\right\}=\widetilde{\Lambda}^{i} .
\end{aligned}
$$

\subsection{Extended Gauge Symmetries}

Now, in order for obtaining the corresponding Lagrangian, we write the generating functional of the topologically massive theory in the fully extended 
phase space as

$$
\begin{aligned}
\mathcal{Z} & =\int \mathcal{D} A^{\mu} \mathcal{D} \pi_{\mu} \mathcal{D} B^{\mu \nu} \mathcal{D} \pi_{\mu \nu} \mathcal{D} \theta \mathcal{D} \pi_{\theta} \mathcal{D} Q^{i} \mathcal{D} P_{i} \mathcal{D} \Phi^{i} \mathcal{D} \Phi^{i j} \\
& \times \delta\left(\widetilde{\varphi}_{\alpha}\right) \delta\left(\Gamma_{\beta}\right) \operatorname{det}\left|\left\{\widetilde{\varphi}_{\alpha}, \Gamma_{\beta}\right\}\right| e^{i S}
\end{aligned}
$$

where

$$
S=\int d^{4} x\left[\pi_{\mu} \dot{A}^{\mu}+\pi_{0 i} \dot{B}^{0 i}+\frac{1}{2} \pi_{i j} \dot{B}^{i j}+\pi_{\theta} \dot{\theta}+P_{i} \dot{Q}^{i}+\frac{1}{2} \epsilon_{i j k} \Phi^{i} \dot{\Phi}^{j k}-\widetilde{\mathcal{H}}_{c}^{\prime}\right],
$$

and $\Gamma_{\alpha}$ are appropriate gauge fixing functions which have non-vanishing Poisson brackets with the modified first-class constraints $\tilde{\varphi}_{\alpha}$.

First, we can easily integrate the momenta variable, $\pi_{0}, \pi_{i}, \pi_{0 i}$, and $\pi_{i j}$ out along with the delta functional, $\delta\left(\widetilde{\pi}_{0}\right), \delta\left(\widetilde{\Omega}_{i}\right), \delta\left(\widetilde{\pi}_{0 i}\right)$, and $\delta\left(\widetilde{\Omega}_{i j}\right)$, respectively. Then, making use of the Fourier transformations of the constraints $\tilde{\Lambda}$, $\tilde{\Lambda}_{i}$ as $\delta(\widetilde{\Lambda})=\int \mathcal{D} \xi \exp \left(-i \int d^{4} x \xi \widetilde{\Lambda}\right), \delta\left(\widetilde{\Lambda}_{i}\right)=\int \mathcal{D} \chi^{i} \exp \left(-i \int d^{4} x \chi^{i} \widetilde{\Lambda}_{i}\right)$, and transforming $A^{0} \rightarrow A^{0}+\xi, B^{0 i} \rightarrow B^{0 i}-\chi^{i}$, and after the Gaussian integrations over $\pi_{\theta}$ and $P_{i}$ variables, we finally obtain the generating functional as

$$
\mathcal{Z}=\int \mathcal{D} A^{\mu} \mathcal{D} B^{\mu \nu} \mathcal{D} \theta \mathcal{D} Q^{i} \mathcal{D} \Phi^{i} \mathcal{D} \Phi^{i j} \mathcal{D} Q^{0} \delta\left(Q^{0}\right) \delta\left(\Gamma_{\beta}\right) \operatorname{det}\left|\left\{\widetilde{\varphi}_{\alpha}, \Gamma_{\beta}\right\}\right| e^{i S_{T}}
$$

where

$$
\begin{aligned}
S_{T} & =\int d^{4} x\left(\mathcal{L}_{S t}+\mathcal{L}_{N W Z}\right) \\
\mathcal{L}_{S t} & =\frac{1}{2}\left(A_{\mu}+\partial_{\mu} \theta\right)^{2}-\frac{1}{4}\left(B_{\mu \nu}-Q_{\mu \nu}\right)^{2}+\frac{1}{2 m} \epsilon_{\mu \nu \rho \sigma}\left(B^{\mu \nu}-Q^{\mu \nu}\right) \partial^{\rho}\left(A^{\sigma}+\partial^{\sigma} \theta\right) \\
\mathcal{L}_{N W Z} & =\left[\left(\partial_{i} A_{0}-\partial_{0} A_{i}\right)-\frac{m}{2} \epsilon_{i j k}\left(B^{j k}-Q^{j k}\right)+\frac{m^{2}}{2} \Phi_{i}\right] \Phi^{i} \\
& -\left[\frac{1}{2} \epsilon_{i j k}\left(A^{i}+\partial^{i} \theta\right)+\frac{1}{m} \partial_{k} B_{0 j}+\frac{1}{2 m} \partial_{0} B_{j k}+\frac{1}{4} \Phi_{i j}\right] \Phi^{j k} \\
& +\frac{1}{2} \epsilon_{i j k} \Phi^{i} \dot{\Phi}^{j k} .
\end{aligned}
$$

Next, let us construct the gauge transformation generator $G$, following Dirac's conjecture [1], for the embedded theory in the standard way,

$$
G=\int d^{4} x \sum_{\alpha} \epsilon^{\alpha} \widetilde{\varphi}_{\alpha}
$$


where $\widetilde{\varphi}_{\alpha}=\left(\widetilde{\pi}_{0}, \widetilde{\Omega}_{i}, \widetilde{\Lambda}, \widetilde{\pi}_{0 i}, \widetilde{\Omega}_{i j}, \widetilde{\Lambda}_{i}\right)$ are the first-class constraints in equation (3.11) in order, and $\epsilon^{\alpha}=\left(\epsilon_{A}^{0}, \epsilon_{A}^{i}, \epsilon_{A}, \epsilon_{B}^{0 i}, \epsilon_{B}^{i j}, \epsilon_{B}^{i}\right)$ are, in general, functions of phase space variables. The infinitesimal gauge transformation for a function $F$ of phase space variables is then given by the relation of $\delta F=\{F, G\}_{D}$, and leads to

$$
\begin{aligned}
\delta A^{0} & =\epsilon_{A}^{0}, & & \delta B^{0 i}=\epsilon_{B}^{0 i}, \\
\delta A^{i} & =\epsilon_{A}^{i}-\partial^{i} \epsilon_{A}, & & \delta B^{i j}=\partial^{i} \epsilon_{B}^{j}-\partial^{j} \epsilon_{B}^{i}+\epsilon_{B}^{i j}-\epsilon_{B}^{j i}, \\
\delta \theta & =\epsilon_{A}, & & \delta Q^{i}=\epsilon_{B}^{i}, \\
\delta \Phi^{i} & =\frac{1}{m} \epsilon^{i j k} \epsilon_{j k}^{B}, & & \delta \Phi^{i j}=-\epsilon^{i j k} \epsilon_{k}^{A} .
\end{aligned}
$$

The above gauge transformation involving the gauge parameters is a symmetry of the Hamiltonian, but not of the Lagrangian. The generator $G$ of the most general local symmetry transformation of a Lagrangian must satisfy the master equation [24]

$$
\frac{\partial G}{\partial t}+\left\{G, H_{T}\right\}=0
$$

which, together with (4.10), implies the following restrictions on the gauge parameters and on the Lagrangian multipliers in the primary Hamiltonian:

$$
\begin{aligned}
\delta v^{\beta} & =\frac{d \epsilon^{\beta}}{d t}-\epsilon^{P}\left(V_{P}^{\beta}+v^{\alpha} C_{\alpha_{P}}^{\beta}\right), \\
0 & =\frac{d \epsilon^{b}}{d t}-\epsilon^{P}\left(V_{P}^{b}+v^{\alpha} C_{\alpha_{P}}^{b}\right) .
\end{aligned}
$$

Here the superscripts $\alpha, \beta,(a, b)$ denote the primary (secondary) constraints, and $V_{Q}^{P}, C_{Q R}^{P}$ are the structure functions of the constrained Hamiltonian dynamics defined by $\left\{H_{c}, \widetilde{\varphi}_{P}\right\}_{D}=V_{P}^{Q} \widetilde{\varphi}_{Q},\left\{\widetilde{\varphi}_{P}, \widetilde{\varphi}_{Q}\right\}=C_{P Q}^{R} \widetilde{\varphi}_{R}$, respectively. From (4.12) we obtain $\epsilon_{A}^{0}=-d \epsilon_{A} / d t$ and $\epsilon_{B}^{0 i}=d \epsilon_{B}^{i} / d t$. Thus, the gauge transformations of $A^{0}$ and $B^{0 i}$ in Eq. (4.10) reduce to

$$
\delta A^{0}=-\frac{d \epsilon_{A}}{d t}, \quad \delta B^{0 i}=\frac{d \epsilon_{B}^{i}}{d t} .
$$

Redefining the gauge parameters [3] as $\bar{\epsilon}_{B}^{i}=\epsilon_{B}^{i}-\partial^{i} \int d t \epsilon_{B}^{0}$ along with the variation $\delta Q^{0}=\epsilon_{B}^{0}$, the final gauge transformations ${ }^{5}$ are nicely summarized

\footnotetext{
${ }^{5}$ We have omitted the bar symbol in the final rules of gauge transformations.
} 
as

$$
\begin{aligned}
\delta A^{\mu} & =-\partial^{\mu} \epsilon_{A}+\delta_{j}^{\mu} \epsilon_{A}^{j}, & & \delta B^{\mu \nu}=\partial^{\mu} \epsilon_{B}^{\nu}-\partial^{\nu} \epsilon_{B}^{\mu}+\left(\epsilon_{B}^{k l}-\epsilon_{B}^{l k}\right) \delta_{k}^{\mu} \delta_{l}^{\nu}, \\
\delta \theta & =\epsilon_{A}, & & \delta Q^{\mu}=\epsilon_{B}^{\mu}, \\
\delta \Phi^{i} & =\frac{1}{m} \epsilon^{i j k} \epsilon_{j k}^{B}, & & \delta \Phi^{i j}=-\epsilon^{i j k} \epsilon_{k}^{A} .
\end{aligned}
$$

Note here that the gauge parameter $\epsilon_{A}$ is related with the topologically massive one-form fields $A^{\mu}$ and their WZ field $\theta$ while the gauge parameters $\epsilon_{B}^{\mu}$ are connected with the two-form fields $B^{\mu \nu}$ and their WZ fields $Q^{\mu}$ resulting in the usual symmetry of the Stückelberg Lagrangian. On the other hand, the gauge parameters $\epsilon_{A}^{i}, \epsilon_{B}^{i j}$ are obtained from the embedded topological constraints $\tilde{\Omega}_{i}, \tilde{\Omega}_{i j}$ with their WZ fields $\Phi^{i}, \Phi^{i j}$ resulting in the new type of WZ Lagrangian. It is easy to check that the total action (4.6) is exactly invariant under those gauge transformation. As results, we have explicitly obtained the usual gauge invariant Stückelberg Lagrangian $\mathcal{L}_{S t}$ for the massive one- and two-form fields, and a new type of the WZ Lagrangian $\mathcal{L}_{N W Z}$ including the WZ fields, $\Phi^{i}, \Phi^{i j}$.

It is appropriate to comment that in the generating functional (4.5) there exists the delta functional of a variable $Q^{0}$ which transforms as $\delta Q^{0}=\epsilon_{B}^{0}$ as shown above. We have introduced this new field to make the final Lagrangian manifestly covariant. Even without this $Q^{0}$ field, we can show that the resulting Lagrangian successfully reproduces all the BFT embedded constraint structure as in the section 3. However, it fails to have manifest covariance. According to the usage of the Hamiltonian formulation, the constraint structure of the Lagrangian (2.1) is called irreducible, in other words, the constraints are linearly independent. On the other hand, the constraint structure of the Stückelberg Lagrangian (4.7) is reducible, i.e., there is a redundant relation among the constraints. In fact, we have introduced the new variable $Q^{0}$ in order for keeping the manifest covariance, while giving up the irreducible property between the constraints.

On the other hand, the new type of the WZ action is related to the symplectic constraints, $\Omega_{i}, \Omega_{i j}$, of the Lagrangian (2.1), which are now converted into the first-class constraints. This seemingly non-covariant form of the Lagrangian $\mathcal{L}_{N W Z}$ comes from the introduction of the auxiliary fields $\Phi^{i}, \Phi^{i j}$ as given by Eq. (3.9) where the distinction between the fields and the momenta is useless. In other words, they look like another Dirac brackets, and in order 
for embedding this symplectic structure fully and getting a completely covariant action such as the symplectic structure free theory, we may introduce infinite numbers of auxiliary fields as discussed in Ref. [27].

\subsection{Various Gauge Fixings}

Now, let us consider various gauge fixings in the path integral (4.3). First, unitary gauge fixings: by fixing unitary gauge means that all the auxiliary fields are set to be zero, $\Phi_{\alpha}=0$, and it is easy task to check that we can recover the original Lagrangian (2.1) in the unitary gauge as follows

$$
\mathcal{Z}=\int \mathcal{D} A^{\mu} \mathcal{D} B^{\mu \nu} e^{i S}
$$

with the action $S$ in Eq. (2.1). Furthermore, it is easy to see that the integration over $B^{\mu \nu}$ yields the Proca action, while the integration over $A^{\mu}$ Kalb-Ramond action. Therefore, we have reconfirmed the well-known result that the action (2.1) is nothing but a master action leading to a dual description, i.e., they have a common origin.

Next, let us consider appropriate gauge fixings showing that the topologically massive theory of first order with the one- and two-form fields is equivalent to the $B \wedge F$ theory. From the generating functional (4.3), we first eliminate the auxiliary fields $\Phi^{i}=0, \Phi^{i j}=0$ by fixing the unitary gauge in part. Then, the resulting Lagrangian is simply the Stückelberg $\mathcal{L}_{S t}$. Next, we consider the following gauge fixings:

$$
\begin{aligned}
\partial_{i} Q^{i} & =0, \\
\partial_{i} B^{0 i} & =0, \\
\chi_{i} & \equiv A_{i}-\frac{1}{m} \epsilon_{i j k} \partial^{j} B^{0 k}=0, \\
\chi_{i j} & \equiv B_{i j}-\frac{1}{m} \epsilon_{i j k} \partial^{k} A^{0}=0 .
\end{aligned}
$$

The momenta, $\pi_{i}, \pi_{i j}, \pi_{0}, \pi_{0 i}$, and $Q^{0}$ fields are integrated out along with the constraints, $\delta\left(\widetilde{\Omega}_{i}\right), \delta\left(\widetilde{\Omega}_{i j}\right), \delta\left(\widetilde{\pi}_{0}\right), \delta\left(\widetilde{\pi}_{0 i}\right)$, and $\delta\left(Q^{0}\right)$, respectively. After the momenta $\pi_{\theta}, P_{i}$, integration along with the constraints $\widetilde{\Lambda}, \widetilde{\Lambda}_{i}$, we obtain the following intermediate generating functional

$$
\mathcal{Z}=\int \mathcal{D} A^{\mu} \mathcal{D} B^{\mu \nu} \mathcal{D} \theta \mathcal{D} Q^{i} \delta\left(\chi_{i}\right) \delta\left(\chi_{i j}\right) \operatorname{det}\left|\left\{\widetilde{\varphi}_{\alpha}, \Gamma_{\beta}\right\}\right| e^{i S},
$$


where the action

$$
S=\int d^{4} x\left[\frac{1}{2 m} \epsilon_{i j k} \dot{A}^{i} B^{j k}-\frac{1}{2 m} \epsilon_{i j k} \partial^{i} B^{j k} \dot{\theta}+\frac{1}{m} \epsilon_{i j k} \dot{Q}^{i} \partial^{j} A^{k}-\widetilde{H}_{c}^{\prime}\right],
$$

and

$$
\widetilde{H}_{c}^{\prime}=\frac{1}{4}\left(B_{i j}-Q_{i j}\right)^{2}-\frac{1}{2}\left(A_{i}+\partial_{i} \theta\right)^{2}+\frac{1}{4 m^{2}} F_{i j} F^{i j}-\frac{1}{2 \cdot 3 ! m^{2}} H_{i j k} H^{i j k} .
$$

Here, we have used identities

$$
\begin{aligned}
& \epsilon_{i j k} \epsilon^{l m n} \partial^{i} B^{j k} \partial_{l} B_{m n}=-\frac{2}{3} H_{i j k} H^{i j k}, \\
& \epsilon_{i j k} \epsilon^{i l m} \partial^{j} A^{k} \partial_{l} A_{m}=\frac{1}{2} F_{i j} F^{i j},
\end{aligned}
$$

with $\partial^{i} A_{i}=0$ which comes from the gauge fixing condition $\partial^{i} \chi_{i}=0$. We have also denoted $H_{i j k}=\partial_{i} B_{j k}+\partial_{j} B_{k i}+\partial_{k} B_{i j}$ and $F_{i j}=\partial_{i} A_{j}-\partial_{j} A_{i}$. The remaining $\theta$ and $Q^{i}$ integrations yields the following action

$$
\begin{aligned}
S=\int d^{4} x & {\left[\frac{1}{2 m} \epsilon_{i j k} \dot{A}^{i} B^{j k}-\frac{1}{4} B_{i j} B^{i j}+\frac{1}{2} A_{i} A^{i}-\frac{1}{4 m^{2}} F_{i j} F^{i j}+\frac{1}{2 \cdot 3 ! m^{2}} H_{i j k} H^{i j k}\right.} \\
& \left.-\frac{1}{8 m^{2}} \epsilon_{i j k} \epsilon^{i l m} \partial^{0} B^{j k} \partial_{0} B_{l m}-\frac{1}{2 m^{2}} \partial_{0} A_{i} \partial^{0} A^{i}\right] .
\end{aligned}
$$

Now, making use of the gauge conditions (4.16) and identities

$$
\begin{aligned}
& -\frac{1}{8 m^{2}} \epsilon_{i j k} \epsilon^{i l m} \partial^{0} B^{j k} \partial_{0} B_{l m}=\frac{1}{4 m^{2}} H_{0 i j} H^{0 i j}-\frac{1}{2 m^{2}} B_{0 i} \nabla^{2} B^{0 i}, \\
& -\frac{1}{2 m^{2}} \partial_{0} A_{i} \partial^{0} A^{i}=-\frac{1}{2 m^{2}} F_{0 i} F^{0 i}+\frac{1}{2 m^{2}} A_{0} \nabla^{2} A^{0},
\end{aligned}
$$

and the equations also obtained from the gauge conditions (4.16)

$$
\begin{aligned}
& \nabla^{2} A^{0}=\frac{m}{2} \epsilon_{i j k} \partial^{i} B^{j k}, \\
& \nabla^{2} B^{0 i}=-m \epsilon^{i j k} \partial^{j} A^{k},
\end{aligned}
$$

we can manipulate the last two terms in the action (4.20) with the others to obtain the well-known $B \wedge F$ theory of

$$
\begin{aligned}
\mathcal{Z} & =\int \mathcal{D} A^{\mu} \mathcal{D} B^{\mu \nu} \operatorname{det}\left|\left\{\widetilde{\varphi}_{\alpha}, \Gamma_{\beta}\right\}\right| e^{i S} \\
S & =\int d^{4} x\left[-\frac{1}{4} F_{\mu \nu} F^{\mu \nu}+\frac{1}{2 \cdot 3 !} H_{\mu \nu \rho} H^{\mu \nu \rho}+\frac{m}{4} \epsilon_{\mu \nu \rho \sigma} B^{\mu \nu} F^{\rho \sigma}\right],
\end{aligned}
$$


where the fields $A^{\mu}, B^{\mu \nu}$ are scaled as $m A^{\mu}, m B^{\mu \nu}$, respectively.

As a result, we have explicitly shown that the equivalence of the topologically massive theory of first order with the one- and two-form fields and the $B \wedge F$ theory on the level of path integral.

\section{Revisit the Gauging of Topologically Mas- sive Theory}

In this section, we will explicitly show the equivalence of gauged massive theory of first order and the Stückelberg Lagrangian, $\mathcal{L}_{S t}$, in which the Lagrangian is obtained from the BFT embedding.

By gauging the fields $A^{\mu}, B^{\mu \nu}$ means the following transformations

$$
A^{\mu} \rightarrow A^{\mu}+\partial^{\mu} \theta, \quad B^{\mu \nu} \rightarrow B^{\mu \nu}-Q^{\mu \nu},
$$

where $Q^{\mu \nu}=\partial^{\mu} Q^{\nu}-\partial^{\nu} Q^{\mu}$. Thus, the gauged massive theory of first order Lagrangian (2.1) is described by

$\mathcal{L}_{G}=-\frac{1}{4}\left(B_{\mu \nu}-Q_{\mu \nu}\right)^{2}+\frac{1}{2}\left(A_{\mu}+\partial_{\mu} \theta\right)^{2}+\frac{1}{2 m} \epsilon_{\mu \nu \rho \sigma}\left(B^{\mu \nu}-Q^{\mu \nu}\right) \partial^{\rho}\left(A^{\sigma}+\partial^{\sigma} \theta\right)$,

which is invariant under the gauge transformations of

$$
\delta A^{\mu}=\partial^{\mu} \epsilon, \quad \delta \theta=-\epsilon, \quad \delta B^{\mu \nu}=\partial^{\mu} \epsilon^{\nu}-\partial^{\nu} \epsilon^{\mu}, \quad \delta Q^{\mu}=\epsilon^{\mu} .
$$

Here, we have redefined the gauge parameters as $\epsilon_{A}=-\epsilon, \epsilon_{B}^{\mu}=\epsilon^{\mu}$ in which

expression is usually seen in the literature and take $\epsilon_{A}^{i}=\epsilon_{B}^{i j}=0$ in the extended gauge symmetries (4.14) in order to focus mainly on the gauging technique. Note that as you already know through the note in the previous sections by taking $\epsilon^{\mu}=\partial^{\mu} \lambda, \delta B^{\mu \nu}$ have vanished clearly indicating that the constraints are not all independent, i.e., reducible.

In order for comparing this action with the Stückelberg Lagrangian $\mathcal{L}_{S t}$ in view of constraint structure, we do partial integration of the terms $G_{0} \dot{\theta}$, $H_{0 i} \dot{Q}^{i}$ to $\dot{G}_{0} \theta,-\dot{H}_{0 i} Q^{i}$, respectively, in the gauged Lagrangian (5.2). Then, the canonical momenta are obtained as

$$
\pi_{0}=-\theta, \quad \pi_{i}=\frac{1}{4 m} \epsilon_{i j k} B^{j k},
$$




$$
\begin{aligned}
\pi_{0 i} & =-Q^{i}, \quad \pi_{i j}=-\frac{1}{2 m} \epsilon_{i j k} A^{k} \\
P_{i} & =Q_{i 0}, \quad P_{0}=0, \quad \pi_{\theta}=\dot{\theta}
\end{aligned}
$$

from which we have the primary constraints as

$$
\begin{aligned}
& \Sigma_{0} \equiv \pi_{0}+\theta \approx 0, \\
& \Sigma_{i} \equiv \pi_{0 i}+Q^{i} \approx 0, \\
& \Omega_{i} \equiv \pi_{i}-\frac{1}{4 m} \epsilon_{i j k} B^{j k} \approx 0, \\
& \Omega_{i j} \equiv \pi_{i j}+\frac{1}{2 m} \epsilon_{i j k} A^{k} \approx 0, \\
& P_{0} \approx 0 .
\end{aligned}
$$

We also get the canonical Hamiltonian as

$$
\begin{aligned}
\mathcal{H}_{c} & =\frac{1}{4}\left(B_{i j}-Q_{i j}\right)^{2}-\frac{1}{2}\left(A_{i}+\partial_{i} \theta\right)^{2}+\frac{1}{2} B_{0 i} B^{0 i}-\frac{1}{2} P_{i} P^{i}-\frac{1}{2} A_{0} A^{0} \\
& +\frac{1}{2} \pi_{\theta}^{2}+\left(P_{i}+B_{0 i}\right) \partial^{i} Q^{0}-\frac{1}{m} \epsilon_{i j k} B^{0 i} \partial^{j} A^{k}-\frac{1}{2 m} A^{0} \epsilon_{i j k} \partial^{i} B^{j k}
\end{aligned}
$$

From the time stability conditions of the primary constraints with the canonical Hamiltonian, there are additional secondary constraints as follows

$$
\begin{aligned}
\Delta & \equiv \frac{d}{d t} P_{0}=\partial^{i}\left(P_{i}+B_{0 i}\right) \approx 0, \\
\Lambda & \equiv \frac{d}{d t} \Sigma_{0}=A_{0}+\frac{1}{2 m} \epsilon_{i j k} \partial^{i} B^{j k}+\pi_{\theta} \approx 0, \\
\Lambda_{i} & \equiv \frac{d}{d t} \Sigma_{i}=-B_{0 i}+\frac{1}{m} \epsilon_{i j k} \partial^{j} A^{k}-P_{i} \approx 0 .
\end{aligned}
$$

It seems appropriate to comment on the linear independence of the constraints. The combination of the above constraints $\Delta, \Lambda_{i}$ vanishes i.e., $\Delta+\partial^{i} \Lambda_{i}=0$, which indicates again that the whole constraints are not linearly independent, i.e., reducible.

Finally, making use of the above constraints, we can obtain the following Hamiltonian

$$
\begin{aligned}
\mathcal{H}_{c} & =\frac{1}{4}\left(B_{i j}-Q_{i j}\right)^{2}-\frac{1}{2}\left(A_{i}+\partial_{i} \theta\right)^{2}+\frac{1}{2}\left(B_{0 i}+P_{i}\right)^{2}-\frac{1}{m} \epsilon_{i j k}\left(B^{0 i}+P^{i}\right) \partial^{j} A^{k} \\
& -\frac{1}{2}\left(A_{0}+\pi_{\theta}\right)^{2}-\frac{1}{2 m}\left(A_{0}+\pi_{\theta}\right) \epsilon_{i j k} \partial^{i} B^{j k}+\pi_{\theta} \Omega+\left(P^{i}+\partial^{i} Q^{0}\right) \Omega_{i} \\
& \approx \widetilde{\mathcal{H}}_{c}^{\prime},
\end{aligned}
$$


where $\widetilde{\mathcal{H}}_{c}^{\prime}$ is given in Eq. (3.13). This shows the equivalence of the gauged Lagrangian (5.2) and the Stückelberg Lagrangian (4.7) on the constraint surface.

\section{Conclusion}

In this paper, we have applied the complete BFT method to the massive theory with one- and two-form fields. We have newly analyzed the full set of constraint structure of the model having no derivatives in the Poisson brackets, which is much simpler than that of the previous work [22] and thus makes it possible to apply the BFT embedding further. Then, we have explicitly carried out the complete BFT embedding of the theory including the gauge symmetry breaking terms and the topological term, which was not gauge invariant. Exploiting the complete BFT embedding we have obtained the gauge invariant Lagrangian corresponding to the first class Hamiltonian, and by identifying the auxiliary fields with the Stückelberg vector fields and new type of WZ fields, we have shown simultaneously the Stückelberg Lagrangian related to the explicit gauge breaking mass term and the new type of WZ action to topological term having novel symmetry. Furthermore, by analyzing the gauged version of the theory we have also shown that the usual Stückelberg Lagrangian is exactly equivalent to the BFT embedded one on the constraint space after taking the unitary gauge $\Phi^{i}=0, \Phi^{i j}=0$.

The work of YWK was supported by the Korea Research Foundation, Grant No. KRF-2002-075-C00007.

\section{References}

[1] P. A. M. Dirac, "Lectures on quantum mechanics" (Belfer graduate School, Yeshiba University Press, New York 1964).

[2] E. S. Fradkin and G. A. Vilkovisky, Phys. Lett. B55, 224 (1975). 
[3] M. Henneaux, Phys. Rept. 126, 1 (1985); See also M. Henneaux and C. Teitelboim, Quantization of gauge systems (Princeton University Press, New Jersey, 1992) and references therein.

[4] C. Becci, A. Rouet and R. Stora, Ann. Phys. [N.Y.] 98, 287 (1976); I. V. Tyutin, Lebedev Preprint 39 (1975); T. Fujiwara, Y. Igarashi and J. Kubo, Nucl. Phys. B341, 695 (1990); Y.-W. Kim, S.-K. Kim, W.T. Kim, Y.-J. Park, K.Y. Kim, and Y. Kim, Phys. Rev. D46, 4574 (1992); R. Banerjee, H. J. Rothe, and K. D. Rothe, Phys. Rev. D49, (1994).

[5] I. A. Batalin and E. S. Fradkin, Phys. Lett. B180, 157 (1986); Nucl. Phys. B279, 514 (1987).

[6] I. A. Batalin and I. V. Tyutin, Int. J. Mod. Phys. A6, 3255 (1991).

[7] N. Banerjee, R. Banerjee and S. Ghosh, Ann. Phys. 241, 235 (1995); Y.-W. Kim, M.-I. Park, Y.-J. Park and S. J. Yoon, Int. J. Mod. Phys. A12, 4217 (1997); R. Banerjee and J. Barcelos-Neto, Nucl. Phys. B499, 453 (1997); M.-I. Park and Y.-J. Park, Int. J. Mod. Phys. A13, 2179 (1998).

[8] R. Banerjee, H.J. Rothe, and K.D. Rothe, Nucl. Phys. B426, 129 (1994); Y.-W. Kim, Y.-J. Park and K.D. Rothe, J. Phys. G24, 953 (1998); Y.W. Kim, Y.-J. Park, and S. J. Yoon, J. Phys. G25, 1783 (1999).

[9] W. Oliveira and J. Ananias Neto, Nucl. Phys. B533, 611 (1998); S.T. Hong, Y.-W. Kim, and Y.-J. Park, Phys. Rev. D59, 114026 (1999); E.M.C. Abreu, J. Ananias Neto, and W. Oliveira, Phys. Lett. B483, 337 (2000); J. Ananias Neto, C. Neves and W. Oliveira, Phys. Rev. D63, 085018 (2001); S.-T. Hong and Y.-J. Park, Phys. Rept. 358, 143 (2002) and references therein; S.M.H. Wong, hep-th/0207194.

[10] N. Banerjee, Subir Ghosh and R. Banerjee, Nucl. Phys. B417, 257 (1994); J. Barcelos-Neto and W. Oliveira, Phys. Rev. D56, 2257 (1997); S.-T. Hong, W.T. Kim, and Y.-J. Park, Phys. Rev. D60, 125005 (1999); S.-T. Hong, Y.-W. Kim, Y.-J. Park, and K. D. Rothe, J. Phys. A36, xxx (2003), hep-th/0210085. 
[11] S.-T. Hong, W.T. Kim, Y.-J. Park and M.-S. Yoon, Phys. Rev. D62, 085010 (2000); R. Amorim and J. Barcelos-Neto, Phys. Rev. D64, 065013 (2001).

[12] R. Banerjee, Phys. Rev. D48, R5467 (1993); W.T. Kim and Y.J. Park, Phys. Lett. B336, 376 (1994); R. Banerjee and H.J. Rothe, Nucl. Phys. B447, 183 (1995); R. Amorim and J. Barcelos-Neto, Phys. Rev. D53, 7129 (1996); Y.-W. Kim and K.D. Rothe, Nucl. Phys. B511, 510 (1998); M. Fleck and H.O. Girotti, Int. J. Mod. Phys. A14, 4287, (1999); A. S. Vytheeswaran Int. J. Mod. Phys. A17, 4095 (2002).

[13] J. Wess and B. Zumino, Phys. Lett. B37, 95 (1971).

[14] R. Banerjee and H. J. Rothe, Nucl. Phys. B447, 183 (1995); Banerjee, H. J. Rothe and K. D. Rothe, Phys. Rev. D55, 6339 (1997).

[15] M. Kalb and P. Ramond, Phys. Rev. D9, 2273 (1974); A. Aurilia and Y. Takahashi, Prog. Theor. Phys. 66, 693 (1981).

[16] S. Deser, R. Jackiw and S. Templeton, Ann. Phys. (N.Y.) 140, 372 (1982).

[17] E. Cremmer and J. Scherk, Nucl. Phys. B72, 117 (1974); T. J. Allen, M. J. Bowick, A. Lahiri, Mod. Phys. Lett. A6, 559 (1991); R. Amorim and J. Barcelos-Neto, Mod. Phys. Lett. A10, 917 (1995); J. Barcelos-Neto and S. Rabello, Z. Phys. C74, 715 (1997).

[18] S. Rey, Phys. Rev. D40, 3396 (1989); E. Poppitz, Nucl. Phys. B542, 31 (1999); L. E. Ibàñez, R. Rabadàn and A. M. Uranga, Nucl. Phys. B542, 112 (1999); D.M. Ghilencea, L.E. Ibàñez, N. Irges, and F. Quevedo, JHEP 08, 016 (2002).

[19] A. Lahiri, Mod. Phys. Lett. A8, 2403 (1993); E. Harikumar and M. Sivakumar, Phys. Rev. D57, 3794 (1998); A. Smailagic and E. Spallucci, Phys. Rev. D61, 067701 (2000).

[20] M. J. Duff. R. Khuri and J. Lu, Phys. Rept. 259, 213 (1995); see also S. E. Hjelmeland and U. Lindström, hep-th/9705122.

[21] N. Banerjee and R. Banerjee, Mod. Phys. Lett. A11, 1919 (1996). 
[22] E. Harikumar and M. Sivakumar, Nucl. Phys. B565, 385 (2000); Int. J. Mod. Phys. A17, 405 (2002).

[23] L. Faddeev and R. Jackiw, Phys. Rev. Lett. 60, 1692 (1985); R. Jackiw, Diverse Topics in Theoretical and Mathematical Physics, (World Scientific, Singapore, 1995).

[24] R. Banerjee, H. J. Rothe, and K. D. Rothe, Phys. Lett. B463, 248 (1999); Phys. Lett. B479, 429 (2000); Soon-Tae Hong, Yong-Wan Kim, Young-Jai Park, and K. D. Rothe, J. Phys. A35, 7461 (2002).

[25] W.T. Kim, Y.-W. Kim, M.-I. Park, Y.-J. Park, and S.J. Yoon, J. Phys. G23, 325 (1997).

[26] Y.-W. Kim, Y.-J. Park, K.Y. Kim, and Y. Kim, Phys. Rev. D51, 2943 (1995).

[27] W.T. Kim, Y.-W. Kim, and Y.-J. Park, J. Phys. A32, 2461 (1999). 